\title{
Intravital and postmortem laboratory diagnostics of hydrophobia clinical cases
}

\author{
Alexander Shabeykin ${ }^{1}$, Alexey Gulyukin ${ }^{1}$, Vladislav Belimenko ${ }^{1, *}$, Tatyana Stepanova ${ }^{1}$, \\ Andrey Kapustin ${ }^{1}$, and Alexey Laishevtcev ${ }^{1}$ \\ ${ }^{1}$ Federal Scientific Centre VIEV" (FSC VIEV), 24/1, Ryazanskiy prospekt, 109428, Moscow, Russia
}

\begin{abstract}
Rabies excitant is a neurophilic virus (Rabiesvirus), regarding to Mononegavirales order, Rhabdoviridae family, Lyssavirus genus. The situation with rabies morbidity in Russia is characterized as extremely unpleasant. Saliva, tear liquid, patient's cornea prints were used in the work as well as, after their death, various segments of brain and salivary glands were used. Additionally, antibody concentration in blood serum of humans passing antirabies therapy after animal bites were investigated. Research Research of eye cornea prints from patient A, suffered from wolf bite, with antibody fluorescent method showed positive result 6 days before the death. The obtained results were confirmed postmortem while the investigation of brain various segments and salivary glands with the use of antibody fluorescent spectroscopy and enzyme-linked analysis method, ELISA, and in biosamples. Intravital research of various biosamples picked from the patient B, suffered from homeless dog bite, showed the precedence of Virus 9 days before the death. Rabies virus antigen was discovered on the basis of research results of eye cornea prints with confocal spectroscopy, saliva samples - with ELISA method, as well as rabies virus genome in saliva and tear liquid was demonstrated with Nested Enzyme-Linked Analysis with Reverse Transcription (Nested RT-PCR). Intravital diagnostics results of hydrophobia patient were confirmed afterwards postmortem while the investigation of brain various segments with confocal fluorescence spectroscopy, ELISA, method and biopsy. Hydrophobia of injured was going on in clinically well-expressed manner. The results of intravital and postmortem laboratory diagnostics of hydrophobia clinical cases in Russian Federation were considered. These cases were the result of wolf and homeless dog bites. Both hydrophobia cases, caused by wild predator and dog bite, were laboratory confirmed yet during patient's life. Introduction of intravital laboratory rabies diagnostics into medical practice is an important element of differential diagnostics.
\end{abstract}

\section{Introduction}

The situation with rabies morbidity in Russia is characterized as extremely unpleasant. For last 20 years the number of animal rabies illnesses alternated from 1,4 to 5,5 thousand, and in average was 3,5 thousand cases per year [1-4].

\footnotetext{
*Corresponding author: vlad_belimenko@mail.ru
} 
Rabies excitant is a neurophilic virus (Rabiesvirus), regarding to Mononegavirales order, Rhabdoviridae family, Lyssavirus genus. Virus genome is not segmented and represents single-rod helically twisted negative RNA. There are five coil proteins in virion: nucleoprotein $(\mathrm{N})$, phosphoprotein $(\mathrm{P})$, matrix protein $(\mathrm{M})$, glycoprotein $(\mathrm{G})$ and RNAdependent-RNA-polymerase (L-large protein) [5, 6].

Rabies is passed through immediate contact way, predominantly via bite, in natural conditions, each new victim is passed the virus via saliva during the bite by rabies contaminated animal which has been already developed acute brain fever with intensive virus replication in brain segments. This specificity of virus predetermines that main kindsreservants of rabies virus in nature - as wild carnivores as well as chiroptera mammalians which can easily deliver a bite according to their behavioral habits and mouth apparatus construction.

Inside kind-reservant population, rabies virus is in stable constant circulation forming border-closed nature epizootic center with cyclic and season disease alternations. The contamination of other animal kinds and human is the embodiment of spillover effect and has a character of accidental self-fading vector.

Epidemiology danger is presented by all known kinds of lyssaviruses, while, the predominant number of hydrophobia cases is connected with rabies virus (RABV). Predators all across the world and bats in North and South America serve as RABV virus reservoir. One of the unique RABV possibilities is referred to its capability to adapt to new kinds-reservants that, while the growth of pet dog population, has lead to appearance of lines of virus transfered to circulation inside this animal type. Proximity of dog to human carries a high epidemiological danger and, at present, dogs are the cause of more than $90 \%$ of hydrophobia illnesses among people.

In Russian Federation, natural type prevails, while wild animals of dog family demonstrate the reservoir. The leading virus species- reservant and agent are foxes, then, on the second place by significance, is a raccoon dog which role in hydrophobia epizootic spread constantly increases in districts with moderate climate $[1,2,3,7,8]$.

Phylogenetic analysis, based on nucleic sequences of gene $\mathrm{N}$ determination, shows the existence of genetic varieties amongst field isolates of hydrophobia virus obtained in different nature-economical zones of the country.

The highest density of disease epizootic centers is referred to 2 territorial clusters covering steppe and forest-steppe zones of the country European part.

Phylogenetic differences between field isolates of rabies virus from the territory of different clusters. shown on Fig.1, support the presence of spatial segmentation of rabies area in Russian Federation that must be obligatory taken into account while anti-epizootic measures $[3,9]$.

In a whole, from 1998 to 2017, on Russia European part, there were registered 56640 cases of hydrophobia among animals [3, 6] Nevertheless, predominance of natural (fox) type of epizootics, also the pursued measures on rabies ictus prevention among dogs and anti-rabies first aid ministration to population in all animal bite cases have allowed to avoid massive cases of human rabies [2-4].

During the same 20-year period, in Russia European part, hydrophobia was registered 186 times in a whole. Together, more than 250 thousand people applied for anti-rabies assistance annually because of the animal bites. Specific anti-rabies treatment includes wound treatment and ant-rabies vaccine injection, in case of medical indication the cure combined course is held: anti-rabies vaccine and anti-rabies immunoglobulin. Standard course of anti-rabies vaccination constitutes 5-6 injections of inactivated concentrated cultural vaccine against rabies that is pursued by stages from 1 st to 90 th day after bite and is interrupted if the data is obtained on that attacking animal was healthy on the moment of bite. 


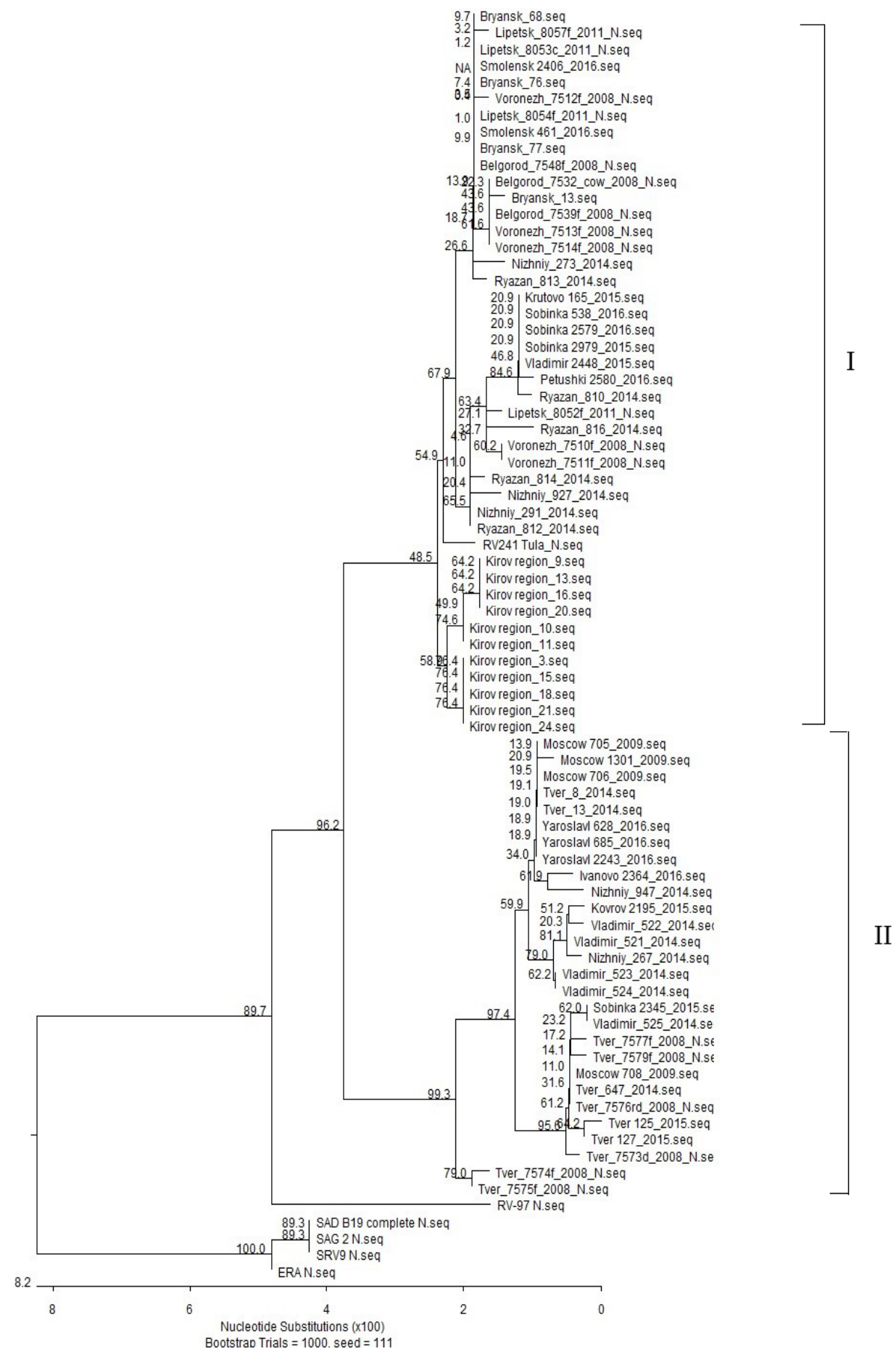

Fig. 1. Phylogenetic dendrogramma obtained on the basis of gene $\mathrm{N}$ fragment of virus field isolates, extracted from the animals from both territorial clusters (I and II) of hydrophobia area on Russian Federation European part.

After transdermal penetration into human or animal organism, rabies virus penetrates 
into the cells of peripheral nervous system. It is considered that, at the place of penetration into organism, rabies virus can replicate in muscle cells as well as virion direct penetration, without preliminary replication, into nervous cells is possible [10-12]. Nevertheless, on all of the early stages of organism contamination, rabies virus uses masking strategy from immune system and his replication stays on minimal level during all of this period. This provides for unique character of rabies infection development that proceeds in the absence of viremia phase during the move into the Central Nervous System (CNS) divisions [13].

Rabies virus penetrates from the place of initial localization into neuron axon via unmyelinated terminal and begins then motion towards CNS with the use of retrograde mechanisms of axoplasmic transport $[14,15]$. Virus particle moving speed across peripheral nervous CNS tissues can alternate but as a rule it is rather small and is from 12 to $24 \mathrm{~mm}$ per day [16]. Neuro invasion period spans the biggest time part of disease development but, during this time, rabies virus is of hard access for the identification by serological/molecular laboratory tests. Hence, it is considered that intravital rabies diagnostics has no practical application. But, hydrophobia intravital diagnostics is necessary for the differentiation from other diseases that are similar according to their clinical features: stupor, tremens, daturine and strychnine poisoning, hysteria. Rabies bulbar forms are differentiated from botulism, poliomyelitis, lethargic cephalitis. Rabies paralytic forms are differentiated from poliomyelitis and infectious polyneuritis. The beginning of hydrophobia clinical exposit is in acute developing brain fever and is accompanied by massive virus replication in different CNS segments. Simultaneously, virus begins spreading intensively eisodically via anterograde axonal transport into different organism parts [12]. From this moment, virus particles can be disclosed by laboratory methods in organism different tissues and liquids such as saliva, smear preparation from eye cornea, skin biopsy samples [17]. Because of quickly flowing terminal stage, express-methods such as fluorescent spectroscopy [11] as well as Reverse Transcription Polymerase Chain Reaction (RT-PCR) [17] are more vital for rabies intravital diagnostics.

The hydrophobia cases caused, in the first case, by wild animal and, in the second one, by dog bite are investigated in the article. The presence of rabies virus was confirmed after the beginning of clinical feature showing-up for both patients with various methods used. This allows to talk about perspective implementation of intravital rabies diagnostics into medical practice.

\section{Materials and methods}

Saliva, tear liquid, patient's cornea prints were used in the work as well as, after their death, various segments of brain and salivary glands were used. Additionally, antibody concentration in blood serum of humans passing antirabies therapy after animal bites were investigated. Laboratory research was held by immediate method of fluorescent antibodies (confocal microscopy), sandwich - variant of enzyme-linked analysis method, ELISA, with the use of rabbit immunoglobulins opposite human globulins and Nested RT-PCR.

\section{Research Results and Discussion}

Hydrophobia for Patient A was developing after wolf bites. The patient had got multiple avulsed wounds of hairy head part, beard area, hand palms, legs. The wolf was shot. wolf body material was brought for expertise, which gave rabies positive result.

After wolf's attack, the injured got combined invasion of anti-rabies immunoglobulin and anti-rabies vaccine first 48 hours, the injured was assigned the standard course of antirabies vaccination but expanded hydrophobia clinics with major psycho-neurological 
symptoms progression acutely developed after 26 days. Hydrophobia and aerophobia had a moderate character; the dominating were physcoviolations and quick development of limb paresis. The death occurred after 7 days.

Patient A was delivered to the hospital next day after rabies clinical features appearance where she was taken the biological material for the investigation. Venous blood was researched on rabies antibodies content, eye cornea prints were investigated on virus antigen.

Simultaneously with injured A, in neighboring region, the attacks of rabies wolf on other 16 people were registered. All were assigned preventing-treating course of anti-rabies vaccines after which blood serum were investigated from 12 injured on determination of the level of specific antibodies by ELISA method and in neutralization reaction (NR).

The blood serum activity of patient A constituted 1:10 in indirect ELISA. Low content of antibodies specific to rabies virus on the background of anti-rabies therapy, probably, was connected with individual specificities of injured immune system as well as complex massive formation between antibodies and the virus which circulate in a very big concentration in organism on last stages of disease development and consequently lowers the determined level of antirabies antibodies.

Antibody titres in ELISA blood serums of injured varied from 1:200 till 1:3200, and, in NR, from 1:163 to 1:397 that was the evidence of good immune defense formation for all the injured.

The investigation of eye cornea prints by direct, confocal fluorescence spectroscopy allowed to discover rabies antigen at proximity to its specific antibodies as a typical distinctly seen bright yellow-green granules of various shape and size with emission intensity in 3 crests (Microphoto 1).

There were no such formations in control samples.

Postmortem analysis of various brain segments by direct fluorescent confocal microscopy gave positive result on rabies virus specific antigen evaluated by crests: in hippocampus - there are multiple specific complexes-inclusions $(+++)$; in cerebral cortex, cerebellum and spinal bulb - there are singular complexes-inclusions $(+++)$.

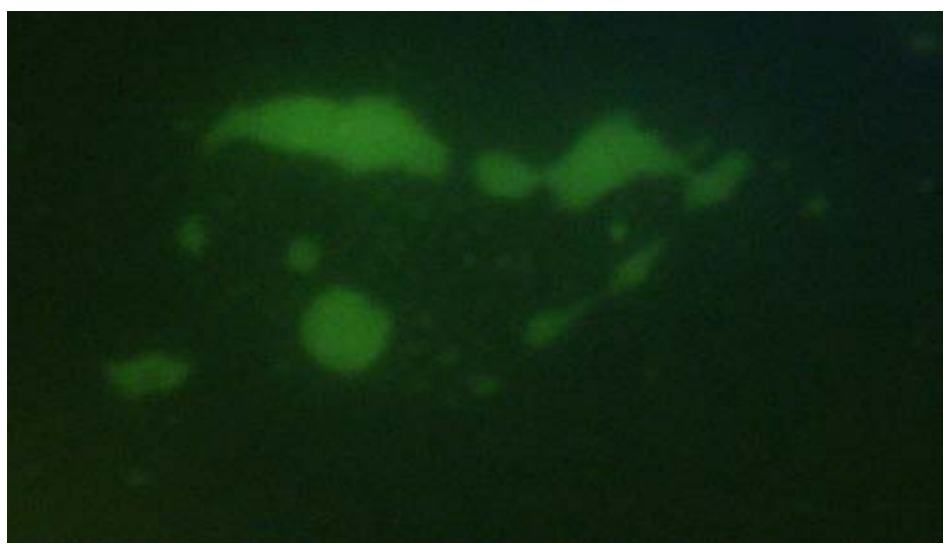

Fig. 2. Intravital discovery of rabies virus antigen in patient A cornea by fluorescence confocal spectroscopy. Zoom is 100 , coefficient is 1.7 .

Rabies virus specific antigen was discovered in suspensions of various brain segments and saliva glands by ELISA method in titres: in hippocampus - 1:160, $\mathrm{K}_{\mathrm{sp}}=2.2$; in spinal bulb - $1: 80, \mathrm{~K}_{\mathrm{sp}}=2.2$;

in cerebellum $-1: 80, K_{\mathrm{sp}}=2.2$; in cerebral cortex $-1: 40, \mathrm{~K}_{\mathrm{sp}}=3.1$; in saliva glands $-1: 20$, $\mathrm{K}_{\mathrm{sp}}=2.1$. Specificity coefficient $\mathrm{K}_{\mathrm{sp}}$ in spectrophotometric registering of ELISA results is more than 2.1 that corresponds to positive respond. 
Babes-Negri bodies were found by light microscopy in prints from hippocampus, spinal bulb. cerebellum and cerebral cortex which are pathognomonic for rabies.

To set the biosample the white suckling mice were used. Intra-cerebral contamination of white mice with brain and saliva gland suspensions caused positive effect on 14-17 days after contamination with emphasized clinical features - hair rumpleness, gibbosity, paralysis and other. Biosample results were maintained by positive results with confocal microscopy of white mice brain prints.

Thus, Patient A hydrophobia, who was bitten by wolf, is supported by laboratory research by: discovery of rabies virus specific antigen in her eye cornea prints by confocal microscopy - 6 days before her death and postmortem - in different brain segments and saliva glands by confocal microscopy, ELISA and light microscopy as well as by abjection of disease excitant via biosample on white mice.

Patient B was delivered to infection hospital on 9th day of illness with hydrophobia features. Earlier, 2 months before hospitalizing, being in South-Eastern Asia, he was bitten by homeless dog into his lower leg. He did not apply for antirabies assistance after the bite.

Patient B hospitalizing was made on $9^{\text {th }}$ day of hydrophobia clinical features. The patient was delivered with behavioral violation features, excitation, tension feeling, breathing difficulties.

Patient B was taken samples for investigations on rabies virus antigen by confocal microscopy, sandwich variant of ELISA method and also by Nested RT-PCR.

The investigation of eye cornea prints of the injured by direct, confocal fluorescence spectroscopy allowed to discover specific rabies antigen, at several localizations as it is seen, as a typical distinctly seen bright yellow-green granules of various shape and size with emission intensity in 3 crests (Microphoto 2 ).

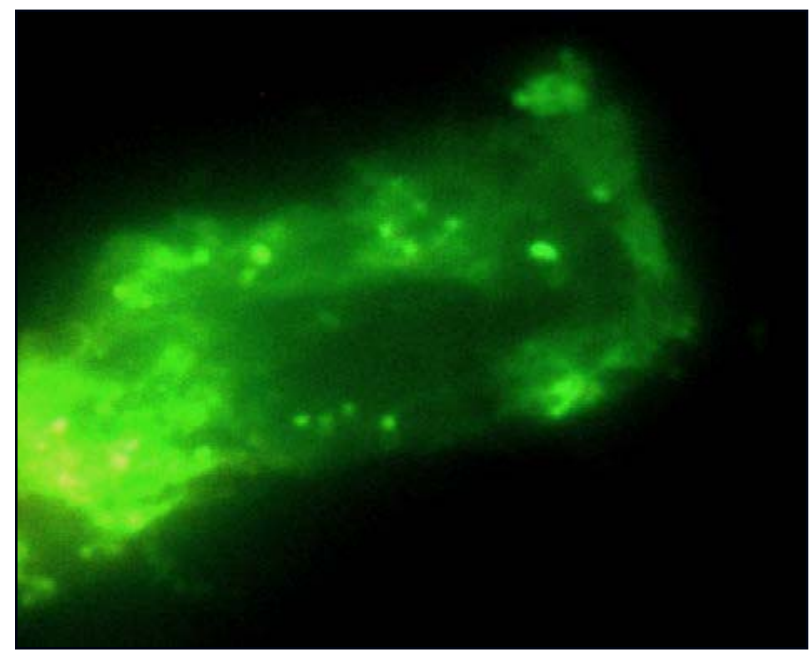

Fig. 3. Intravital discovery of rabies virus antigen in patient $\mathrm{B}$ cornea by fluorescence confocal spectroscopy, Zoom is 100 , coefficient is 1.7 .

There were no such formations in control samples.

Rabies virus antigen with titre $1: 16, \mathrm{~K}_{\mathrm{sp}}=2,2$ was discovered in investigation of patient saliva sample by ELISA method.

Rabies virus genome was distinguished by Nested RT-PCR in saliva and tear samples of Patient B (Microphoto 3). 


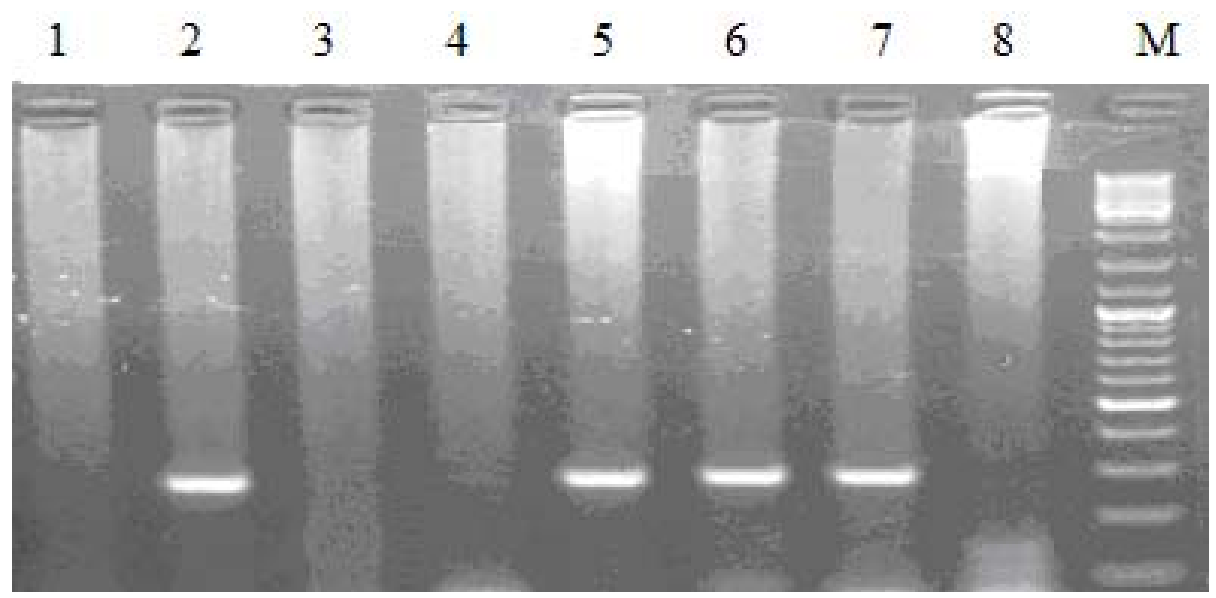

Fig. 4. Electrophoresis of second stage products of amplification (fragment 288 n.p.). Labels: 1negative control sample; 2 - tear liquid of Patient B with clinical show of hydrophobia; 3 - tear liquid of healthy human; 4- healthy person saliva; 5- rabies virus, industrial strain "Sheep" GNKI;6- rabies standard virus CVS; 7- Patient B saliva, with clinical rabies exposition; 8- healthy person saliva; Mmolecular weight marker of 100-3000 nucleotide pairs (Ferments).

Thus, intravital diagnosis - hydrophobia - was confirmed by various laboratory methods. On the 9th day of disease clinical flow, rabies virus antigen was being distinguished in prints from eye cornea and in saliva samples and rabies virus genome was being discovered in saliva samples and tears of the patient.

Patient B passed away on clinical stage day 18 , being in coma.

Postmortem analysis of brain various segments samples (hippocampus, spinal bulb, cerebellum, cerebral cortex) by confocal microscopy and ELISA gave positive result on discovery of the specific antigen of rabies virus. Biopsy on white mice also gave positive result

Thus. hydrophobia was laboratory confirmed by positive diagnosis while eye cornea prints investigation of the patient with confocal microscopy, of saliva by ELISA, as well as of saliva and tear by RT_PCR 9 days before patient's death.

\section{Conclusions}

1. Patient A hydrophobia, who was bitten by wolf, was established for the injured when she was alive: it was done by discovery of rabies virus specific antigen in eye cornea prints with confocal microscopy - 6 days before patient death and confirmed postmortem - rabies antibody was discovered in different brain segments and saliva glands.

2. Patient A had incubation period of 27 days. Patient death had occurred on illness 7 th day. The illness and the death of hydrophobia patient A was the consequence of multiple, deep bites of dangerous localization (head, face, neck, hand palm), caused by rabid wolf.

3. Patient B hydrophobia, bitten by homeless dog, was established during the life of injured: by the discovery rabies virus specific antigen in eye cornea prints with confocal microscopy, in saliva - by ELISA, of rabies virus genome in saliva and tear liquid - with RT-PCR 9 days before the patient death and was confirmed postmortem - in different brain segments with confocal microscopy, ELISA as well as with disease excitant extraction with biopsy on white mice. The obtained results confirm diagnosis made clinically hydrophobia.

4. Injured B incubation period constituted 55 days. Patient death had occurred on illness 18th day. The illness and death of patient $B$ due to hydrophobia was a result of injured did 
not apply for antirabies assistance.

5. Both hydrophobia cases, caused by wild predator and dog bite, were laboratory confirmed yet during patient's life. Introduction of intravital laboratory rabies diagnostics into medical practice is an important element of differential diagnostics.

\section{Acknowledgements}

Financing: The research support was made with the help of state budget task 0578-20190003. Interest conflict: The authors have declared conflict interest lack.

\section{References}

1. B. Dietzschold, J. Li, M. Faber, M. Schnell, Future Virol. 3(5), 481-490 (2008)

2. A.M. Gulyukin, Voprosy virusologii 59(3), 5-10 (2014)

3. R.V. Herwijnen, A.A. Shabeykin, A.M. Gulyukin, Veterinary and feeding 2, 19-23 (2015)

4. A.V. Ivanov, N.A. Khismatullina, A.M. Gulyukin, Veterinary doctor 4, 4-6 (2010)

5. K.J. Koch, J.W. Sagartz, D. Davidson, K. Lawhaswahs, Am. J. Clin. Pathol. 63, 509515 (1975)

6. L. Dacheux, Clin Infect Dis. 47, 1410-17 (2008)

7. C.I. Macedo, P.J. Carnieli, P.E. Brandao, Braz. J. Infect. Dis. 10, 341-345 (2006)

8. A. Mahadevan, M.S. Suja, R.S. Mani, S.K. Shankar, Neurotherapeutics 13(3), 477-492 (2016)

9. R.S. Mani, S.N. Madhusudana, Scientific World Journal 2, 569-712 (2013)

10. F.A. Murphy, Arch Virol. 54, 279-297 (1977)

11. E.G. Simonova, K.Y. Zubareva, S.A. Kartavaya et al, Infektsionnye Bolezni 16(3), 31 36 (2018) DOI: 10.20953/1729-9225-2018-3-31-36

12. M.A. Svotina, G.G. Absatirov, M.S. Shalmenov et al., Biology and Medicine 7(5), 1-3 (2015)

13. A.A. Shabeykin, A.M. Gulyukin, Russian Veterinary 4, 5-7 (2015)

14. A.M. Gulyukin, A.A. Shabeykin, V.V. Belimenko et al., IOP Conf. Ser.: Earth Environ. Sci. 421, 052017 (2020) https://doi.org/10.1088/1755-1315/421/5/052017

15. Expert Consultation on Rabies. 1. Report. Rep. Series 931 (WHO Tech., Geneva, 2004)

16. I. Zimmermann, Tierarztl. Eachr. 84(9), 172-4 (1971)

17. https://who-rabies-bulletin.org/

18. https://talk.ictvonline.org/taxonomy/ 